\title{
Fatores associados à violência física grave contra crianças e adolescentes com transtornos mentais
}

\author{
Factors associated with severe physical violence against children and adolescents with mental disorders
}

Factores asociados a la violencia física grave contra niños y adolescentes con trastornos mentales

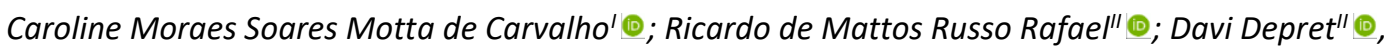 \\ Anna Tereza Miranda Soares de Moura ${ }^{\prime \prime \prime}{ }_{\odot}$; Mercedes Neto ${ }^{\prime \prime}$; Pedro Moacyr Chagas Brandão Junior ${ }^{\prime \prime \prime} \oplus_{\bullet}$ \\ 'Universidade Estácio de Sá, Rio de Janeiro, RJ, Brasil; "Universidade do Estado do Rio de Janeiro, Rio de Janeiro, RJ, Brasil; \\ 'I'Universidade de Vassouras, Vassouras, RJ, Brasil
}

\begin{abstract}
RESUMO
Objetivo: analisar os fatores associados à ocorrência de violência física grave em crianças e adolescentes com transtornos mentais. Método: estudo transversal com 274 pacientes atendidos em uma unidade de atenção psicossocial de Nova Iguaçu, entre outubro e dezembro de 2016. Além de dados sociodemográficos e clínicos dos cuidadores e crianças, a violência familiar foi apreendida pelo "Parent-Child Conflict Tactics Scales". Resultados: o tempo de atendimento na unidade de saúde combinada com a jornada semanal de cuidado pelo cuidador resultaram em altas chances de ocorrência de violência física grave (OR 5,0; $p$-valor 0,002$)$. Por outro lado, a participação em programas de transferência de renda $(O R$ 0,5; $p$-valor 0,015$)$ demonstrou proteção das crianças e adolescentes às violências. Conclusão: as características sociodemográficas e clínicas parecem estar relacionadas à ocorrência de maus-tratos físicos. Para prevenir episódios, principalmente devido à sobrecarga dos cuidadores, parece imprescindível que as famílias sejam inseridas no cotidiano do cuidado mental.

Descritores: Criança; Adolescente; Transtornos Mentais; Violência.
\end{abstract}

\begin{abstract}
Objective: to analyze factors associated with the occurrence of severe physical violence in children and adolescents with mental disorders. Method: cross-sectional study with 274 patients attending a psychosocial care unit in Nova Iguaçu between October and December 2016. In addition to sociodemographic and clinical data on caregivers and children, family violence was measured by the "Parent-Child Conflict Tactics Scales". Results: The time spent in the health unit combined with the week's schedule of care by the caregiver resulted in high chances of serious physical abuse (OR 5.0; $p$-value 0.002). On the other hand, participation in cash transfer programs (OR 0.5, p-value 0.015 ) was found to protect children and adolescents from violence. Conclusion: sociodemographic and clinical characteristics seem to be related to the occurrence of physical abuse. To prevent such episodes, it seems essential to include families in daily mental care, mainly because caregivers are overloaded.
\end{abstract}

Descriptors: Child; Adolescent; Mental Disorders; Violence.

\section{RESUMEN}

Objetivo: analizar los factores asociados a la ocurrencia de violencia física grave en niños y adolescentes con trastornos mentales. Método: estudio transversal junto a 274 pacientes atendidos en una unidad de atención psicosocial en Nova Iguaçu, entre octubre y diciembre de 2016. Además de los datos sociodemográficos y clínicos sobre los cuidadores y los niños, la violencia familiar fue medida por las "Parent-Child Conflict Tactics Scales". Resultados: El tiempo de permanencia en la unidad de salud combinado con la jornada semanal de cuidado por parte del cuidador resultaron en altas probabilidades de violencia física grave $(O R 5,0 ; p$-valor 0,002). Por otro lado, la participación en programas de transferencia de ingresos $(O R$ 0,5; $p$-valor $0,015)$ demostró protección de los niños y adolescentes contra la violencia. Conclusión: las características sociodemográficas y clínicas parecen estar relacionadas con la ocurrencia de maltratos físicos. Para prevenir episodios, principalmente derivados de la sobrecarga de los cuidadores, parece ser fundamental que se incluyan las familias en el cotidiano de cuidado mental. Descriptores: Niño; Adolescente; Trastornos Mentales; Violencia.

\section{INTRODUÇÃO}

O interesse por estudos sobre experiências adversas na infância e adolescência tem crescido no Brasil e no mundo, incluindo uma produção importante sobre abuso infantil, uma vez que sua aceitação é influenciada por hábitos sociais, valores, crenças e julgamentos ${ }^{1-5}$. Embora sua classificação seja vasta e às vezes divergente, as noções propostas pelo Relatório Mundial sobre Violência e Saúde ${ }^{6}$ para definição de abuso e violência foram adotados na maior parte das pesquisas nacionais e internacionais. Com base nessa classificação, a violência é entendida como o uso intencional de poder ou força física com potencial dano ou prejuízo para quem sofre e vivencia esses atos. Adicionalmente, as violências graves são aquelas com potencial de causar danos diretos e, em geral, utilizam táticas de poder envolvendo armas brancas ou de fogo $0^{7,8}$.

Autor correspondente: Ricardo de Mattos Russo Rafael, E-mail: prof.ricardomattos@gmail.com Editora Científica: Cristiane Helena Gallasch; Editora Associada: Sonia Acioli Oliveira. 
Ao contrário do que se espera para o ambiente doméstico, muitos autores têm demostrado que é justamente neste espaço que as violências são perpetradas e de alguma forma ocultadas, principalmente ao se considerar a dificuldade dos setores públicos em abordar tais situações em ambiente privado ${ }^{9}$. Estudos realizados no Brasil apontam para uma magnitude da violência contra crianças e adolescentes bastante variável, que aparentemente se agrava quando são acometidos por condições crônicas, como os transtornos mentais ${ }^{10-12}$. Acredita-se que crianças com transtornos mentais graves apresentam limitações que demandam cuidados intensos e especiais, exigindo mudanças na dinâmica familiar e, consequentemente, aumentando as chances de violências físicas.

Alguns aspectos devem ser destacados, pois, embora existam muitos movimentos legais e iniciativas de saúde para limitar as violências no ambiente domiciliar, uma parte significativa das crianças é educada a partir de experiências abusivas. A educação baseada no castigo corporal ainda faz parte do cotidiano de muitas famílias brasileiras ${ }^{13,14}$. Apesar de todas as mudanças substanciais no contexto político e social, a visão tradicional dos papéis de gênero, que centraliza a responsabilidade de cuidar de crianças e adolescentes quase que exclusivamente nas mulheres, ainda se mantém e talvez seja uma possível causa de sobrecarga materna ${ }^{15}$.

Neste sentido, acreditando que o desvelamento das principais características associadas às violências possa auxiliar no desenvolvimento de políticas públicas e estratégias de atenção mais integral a este subgrupo populacional, este artigo tem como objetivo analisar os fatores associados à ocorrência de violências físicas graves em crianças e adolescentes com transtorno mental.

\section{MÉTODO}

Trata-se de um estudo transversal ancorado no projeto "Violência Familiar em Crianças e Adolescentes com Transtornos Mentais: reflexões a partir da Saúde Mental e da Atenção Básica", realizado com um conjunto de pais e cuidadores de pacientes atendidos em uma unidade de atenção psicossocial de Nova Iguaçu (Rio de Janeiro) no período de outubro a dezembro de 2016.

A população de estudo foi composta por responsáveis de crianças e adolescentes de até 18 anos, residentes em Nova Iguaçu (RJ) e atendidos na unidade nos últimos 12 meses. Considerando a complexidade da confirmação ou suspeita do diagnóstico de transtorno mental grave na infância, foram excluídos os pacientes que compareceram somente à primeira consulta e aqueles que não possuíam plano terapêutico que indicasse acompanhamento no serviço de saúde. Das 340 crianças e adolescentes cadastrados na unidade, 38 foram excluídos pelos critérios acima. Houve 9,3\% (n=28) de recusa dos pais e responsáveis dos pacientes elegíveis, sendo a amostra final composta por 274 participantes.

Os dados foram coletados por meio de um questionário estruturado com aspectos da doença e do cuidado à criança e ao adolescente com transtorno mental dividido em três módulos. O primeiro módulo foi composto por variáveis sociodemográficas de pais, cuidadores, e das crianças e adolescentes. As variáveis demográficas dos pais (idade, raça/cor, escolaridade, estado civil e participação em programas de transferência de renda) foram mensuradas por itens da Pesquisa Nacional por Amostra de Domicílios - PNAD ${ }^{16}$.

As variáveis socioeconômicas foram avaliadas por meio do instrumento "Critérios Brasil" ${ }^{17}$ considerando bens e serviços contratados pela família, estrutura da casa e escolaridade do chefe de família para classificar os indivíduos nos seguintes estratos econômicos: A (renda média domiciliar: R\$ 20.888,00); B1 (renda familiar média: R\$9.254,00); B2 (renda familiar média: R\$ 4,852,00); C1 (renda familiar média: R\$2.705,00); C2 (média de R\$ 1.625,00); D-E (renda familiar média: $R \$ 768,00)$. Os dados dos estratos "B1" e "B2" foram agregados para formar um único estrato denominado "B". A mesma técnica foi aplicada nos estratos "C1" e "C2". A segunda parte foi composta por questões sobre idade e cor / raça da pele de crianças e adolescentes.

O segundo módulo contemplou dados clínicos e de assistência à saúde de crianças e adolescentes e composto pelas seguintes variáveis: relação do cuidador principal (coletado no item: quem é a pessoa que mais tempo passa cuidando da criança / adolescente?); tempo de atendimento (em anos) desde o diagnóstico; jornada semanal dedicada ao cuidado (tempo em horas); e tempo de acompanhamento na unidade de atenção psicossocial.

O módulo final coletou informações sobre a violência física grave perpetrada por familiares/cuidadores contra criança ou adolescente, mensurada por meio do "The Parent-Child Conflict Tactics Scales" (CTSPC), validado e adaptado transculturalmente para uso no Brasil [18]. Com base na Teoria dos Conflitos entre pais e filhos, o CTSPC tem 22 itens que medem a disciplina não violenta, agressão psicológica, violência física e violência física grave ${ }^{18}$. Este trabalho 
analisou a violência físico grave como variável dependente, considerando violência positiva quando pelo menos um item da escala apresentou positividade.

O processamento estatístico foi realizado no software Stata SE $15^{19}$, inicialmente com análises univariadas para reconhecer a distribuição dos dados e descrever o perfil da amostra. Foram realizadas análises bivariadas, calculandose os Odds Ratio (OR) e os respectivos Intervalos de Confiança a 95\% (IC95\%), aplicando-se o Teste Qui-quadrado. As variáveis com $\mathrm{p}$-valor até 0,20 foram incluídas no modelo inicial de análise múltipla.

A análise de regressão logística com técnica stepwise backward manual foi realizada para detectar os fatores associados ao desfecho e controlar os fatores de confusão, removendo individualmente as covariáveis não significativas até chegar a um modelo final (todas as variáveis com $p$-valor $<0,05$ ). Todas as interações entre as variáveis independentes e o desfecho foram testadas.

A pesquisa foi conduzida de acordo com os preceitos éticos envolvendo seres humanos, de acordo com a legislação internacional e brasileira e foi aprovada pelo Comitê de Ética em Pesquisa (Parecer de Aprovação no 1.789.738). O Termo de Consentimento Livre e Esclarecido foi assinado por todos os participantes da investigação. $\mathrm{O}$ anonimato, a confidencialidade e a redução de potenciais danos foram garantidos ao longo do processo de coleta de dados, realizando as entrevistas em ambiente privativo e acompanhando as famílias nos momentos subsequentes à coleta de dados.

\section{RESULTADOS}

A média de idade dos participantes do estudo foi de 38,4 anos (DP 8,9), com 83,5\% acima de 30 anos. Os cuidadores primários eram mulheres $(86,1 \%)$, pretas e pardas $(73,3 \%)$, com mais de 8 anos de estudo $(57,8 \%)$, com companheiro (52,0\%), cuja ocupação estava ligada ao trabalho doméstico ou que estavam desempregadas $(57,1 \%)$, e pertencentes às classes econômicas $C(46,7 \%)$ e $D / E(43,1 \%)$. Eles receberam alguma forma de ajuda financeira do Estado em programas de transferência de renda (69,3\%). A mãe foi identificada como cuidadora principal em $77,1 \%$ dos casos e possuíam ocupações relacionadas ao trabalho doméstico ou desempregadas (65,6\%). Em relação ao tempo de cuidado, 55,3\% dos participantes tinham uma Jornada Semanal de Cuidado mais de 40 horas semanais, a partir de mais de 10 anos $(48,7 \%)$.

Em relação às características sociodemográficas das crianças e adolescentes atendidos, observou-se que 54,8\% tinham entre 12 e 18 anos; em média 11,2 anos (DP 3,7). Eram predominantemente do sexo masculino $(63,9 \%)$ e da cor preta e parda (63,5\%). Em média, crianças e adolescentes foram diagnosticados 6,1 anos antes (DP 4,3); 74,6\% com menos de 10 anos; e possuíam 1,5 ano (DP 1,87) de acompanhamento na unidade de saúde (cenário do estudo).

A Tabela 1 mostra os resultados da análise bivariada entre violência física grave contra crianças e adolescentes de acordo com as covariáveis referentes às características do cuidador e das crianças e adolescentes.

Relações estatisticamente significativas foram observadas com a faixa etária, gênero, inscrição em programas de transferência de renda, tempo de atendimento e jornada semanal de cuidado pelo cuidador. Já em relação as características das crianças e adolescentes, a violência física grave teve relação estatisticamente significativa com a faixa etária e o tempo de diagnóstico.

A Tabela 2 apresenta os resultados da análise múltipla por regressão logística para violência física grave.

Vale ressaltar que o apoio financeiro do governo (OR ajustado 0,5; p-valor 0,026) mostrou uma associação protetora contra abuso físico grave. Por outro lado, a idade de 31 a 40 anos, o sexo do cuidador, o tempo de cuidado no serviço, a Jornada Semanal de Cuidado e o tempo de diagnóstico produziram efeitos sobre a ocorrência de violência física grave.

A Tabela 3 apresenta os resultados das análises de regressão logística para testar as interações entre as variáveis independentes e o desfecho.

Embora todas as variáveis do modelo tenham sido testadas, a interação foi observada apenas entre o tempo de atendimento e a Jornada Semanal de Cuidado. 
TABELA 1: Associação entre violência física grave e as características dos cuidadores, das crianças e dos adolescentes com transtornos mentais. Nova Iguaçu, Rio de Janeiro, Brasil, 2016

\begin{tabular}{|c|c|c|}
\hline Variáveis & OR bruto (IC95\%) & p-valor \\
\hline \multicolumn{3}{|l|}{ Relacionadas aos cuidadores } \\
\hline \multicolumn{3}{|l|}{ Faixa etária } \\
\hline 18 a 30 anos & 1 & \\
\hline 31 a 40 anos & $2,5(1,2-5.2)$ & 0,012 \\
\hline 41 a 50 anos & $1,7(0,7-4.1)$ & 0,196 \\
\hline Maior que 50 anos & $1,6(0,6-4,1)$ & 0,332 \\
\hline \multicolumn{3}{|l|}{ Sexo } \\
\hline Feminino & 1 & - \\
\hline Masculino & $3,3(1,6-6,8)$ & 0,001 \\
\hline \multicolumn{3}{|l|}{ Raça/Cor } \\
\hline Branca & 1 & \\
\hline Preta/Para & $0,7(0,4-1.3)$ & 0,271 \\
\hline Amarela/Indígena & $1,6(0,5-4.8)$ & 0,378 \\
\hline \multicolumn{3}{|l|}{ Escolaridade } \\
\hline Até 8 anos & 1 & \\
\hline Maior que 8 anos & $0,9(0,6-1,5)$ & 0,813 \\
\hline \multicolumn{3}{|l|}{ Status conjugal } \\
\hline Com parceiro & 1 & \\
\hline Sem parceiro & $0,9(0,6-1,5)$ & 0,699 \\
\hline \multicolumn{3}{|l|}{ Ocupação } \\
\hline Outras & 1 & \\
\hline Do lar/Desempregado & $1,2(0,7-1,9)$ & 0,474 \\
\hline \multicolumn{3}{|l|}{ Classe econômica } \\
\hline$A / B$ & 1 & \\
\hline C & $1,4(0,6-3,2)$ & 0,405 \\
\hline $\mathrm{D} / \mathrm{E}$ & $0,7(0,3-1,7)$ & 0,475 \\
\hline \multicolumn{3}{|c|}{ Programa de transferência de renda } \\
\hline No & 1 & \\
\hline Yes & $0,5(0,3-0,9)$ & 0,015 \\
\hline \multicolumn{3}{|l|}{ Cuidador principal: mãe } \\
\hline No & 1 & \\
\hline Yes & $0,8(0,4-1,3)$ & 0,344 \\
\hline \multicolumn{3}{|l|}{ Tempo de cuidado no serviço } \\
\hline Até 10 anos & 1 & \\
\hline Mais que 10 anos & $2,5(1,5-4,0)$ & $<0,001$ \\
\hline \multicolumn{3}{|c|}{ Jornada seminal de cuidado (maior que $40 \mathrm{~h}$ ) } \\
\hline Não & 1 & \\
\hline $\operatorname{Sim}$ & $1,6(1,1-2,6)$ & 0,048 \\
\hline \multicolumn{3}{|c|}{ Relacionadas as crianças e adolescentes } \\
\hline \multicolumn{3}{|c|}{ Faixa etária } \\
\hline 12 a 18 anos & 1 & \\
\hline Até 11 anos & $0,6(0,4-1,0)$ & 0,034 \\
\hline \multicolumn{3}{|l|}{ Sexo } \\
\hline Feminino & 1 & \\
\hline Masculino & $0,9(0,6-1,5)$ & 0,871 \\
\hline \multicolumn{3}{|l|}{ Raça/cor } \\
\hline Branca & 1 & \\
\hline Preta/Parda & $1,1(0,6-2,0)$ & 0,624 \\
\hline Amarela/Indígena & $1,7(0,7-4,1)$ & 0,231 \\
\hline \multicolumn{3}{|l|}{ Tempo de diagnóstico } \\
\hline Até 4 anos & 1 & \\
\hline De 5 a 9 anos & $1,7(0,9-2,9)$ & 0,082 \\
\hline 10 anos ou mais & $1,6(0,9-2,9)$ & 0,124 \\
\hline
\end{tabular}


TABELA 2: Odds Ratio ajustado (modelo final) entre as variáveis independentes e a violência física grave. Nova Iguaçu, Rio de Janeiro, Brasil, 2016.

\begin{tabular}{|c|c|c|}
\hline Variáveis & OR ajustado (IC95\%) & p-valor \\
\hline \multicolumn{3}{|l|}{ Idade do cuidador } \\
\hline 18 a 30 anos & 1 & - \\
\hline 31 a 40 anos & $2,4(1,0-5,5)$ & 0,039 \\
\hline 31 a 50 anos & $1,2(0,4-3,3)$ & 0,704 \\
\hline Maior que 50 anos & $0,9(0,3-2,7)$ & 0,814 \\
\hline \multicolumn{3}{|l|}{ Sexo do cuidador } \\
\hline Feminino & 1 & \\
\hline Masculino & $5,7(2,2-14,6)$ & $<0,001$ \\
\hline \multicolumn{3}{|c|}{ Programa de transferência de renda } \\
\hline No & 1 & \\
\hline Sim & $0,5(0,3-0,9)$ & 0,026 \\
\hline \multicolumn{3}{|c|}{ Tempo de cuidado (em anos) } \\
\hline Até 10 anos & 1 & - \\
\hline Maior que 10 anos & $2,9(1,2-7,2)$ & 0,018 \\
\hline \multicolumn{3}{|c|}{ Jornada seminal de cuidado (maior que $40 \mathrm{~h}$ ) } \\
\hline Não & 1 & \\
\hline Sim & $1,5(1,1-2,3)$ & 0,047 \\
\hline \multicolumn{3}{|c|}{ Tempo de diagnóstico } \\
\hline Até 4 anos & 1 & - \\
\hline De 5 a 9 anos & $2,1(1,1-4,0)$ & 0,024 \\
\hline 10 anos ou mais & $1,3(0,6-2,8)$ & 0,454 \\
\hline
\end{tabular}

TABELA 3: Análise de regressão logística para testagem de interação para violência física grave contra crianças e adolescentes em função do tempo (> 10 anos) e da jornada semanal (> 40 horas) de cuidados. Nova Iguaçu, Rio de Janeiro, Brasil, 2016.

\begin{tabular}{lcc}
\hline Variables & OR ajustado (IC95\%) & p-value \\
\hline Tempo de cuidado $\leq 10$ anos \& Jornada Semanal de Cuidado $\leq 40$ horas & 1 & - \\
Tempo de cuidado $\leq 10$ anos \& Jornada Semanal de Cuidado $>$ 40 horas & $1,5(0,7-3,4)$ & 0,297 \\
Tempo de cuidado $>10$ anos \& Jornada Semanal de Cuidado $\leq 40$ horas & $2,4(0,8-6,6)$ & 0,096 \\
Tempo de cuidado $>10$ anos \& Jornada Semanal de Cuidado $>$ 40 horas & $5,0(1,8-13,9)$ & $\mathbf{0 , 0 0 2}$ \\
\hline
\end{tabular}

Legenda: OR ajustado pelas variáveis idade e sexo do cuidador, inscrição em programa de transferência de renda, tempo de cuidado (em anos), jornada semanal de cuidado e tempo de diagnóstico.

\section{DISCUSSÃO}

Os principais resultados apontam para a importância do apoio aos cuidadores de crianças e adolescentes com transtornos mentais graves, sobretudo ao considerar que estes casos representam condições crônicas com alto potencial de dependência. A clássica coorte de Illinois com 101.189 inscritos do Medicaid, nascidos entre 1990 e 1996, apontou que as condições crônicas, especialmente os transtornos mentais, tendem a dobrar o risco (RR 2,0; IC 95\%: 1,7 $/ 2,4)$ de violência física ${ }^{20}$. Outros estudos também reforçam os efeitos da sobrecarga das famílias como possíveis elementos na causalidade das violências ${ }^{21,22}$, sobretudo pelos potenciais efeitos do adoecimento sobre a dinâmica familiar.

Estes resultados coadunam os achados do presente estudo, que revelou maiores chances de violência física grave associadas aos longos períodos (em anos) e jornadas semanais de cuidado superiores a 40 horas. Ou seja, foi identificado que quanto maior o tempo de cuidado dedicado às crianças e adolescentes, maiores as chances de violência física grave, demostrando os estressores cotidianos relacionados ao tempo de cuidado como proxy da sobrecarga dos cuidadores.

Não é fato novo que as condições crônicas produzem mudanças na vida da família, exigindo ajustes na organização do dia a dia para o atendimento às necessidades da pessoa sob cuidados ${ }^{23}$. 0 desequilíbrio e a deterioração da dinâmica familiar são eventos comuns e intimamente associados à ocorrência de conflitos em decorrência da elevada carga de demandas ${ }^{24}$. $O$ estresse associado a eventos adaptativos pode causar sofrimento ao cuidador, aumentando as chances de ocorrência de conflitos e, como consequência, de violências a quem está sendo cuidado ${ }^{10}$, especialmente frente a ausência de dinâmicas que produzam o necessário suporte familiar ${ }^{25,26}$. 
Crianças com transtornos mentais apresentam alto risco de violência física desde as menores idades, aumentando as chances de consequências graves no desenvolvimento físico e emocional ao longo da vida, pois o abuso tende a se repetir com o tempo ${ }^{20}$ produzindo marcas de difícil mensuração. É importante lembrar que o castigo corporal ainda é o cerne da educação e da resolução de conflitos envolvendo pais e filhos, aspectos que podem favorecer a ocorrência de violências frente à sobrecarga dos cuidadores e à falta de suporte familiar ${ }^{27}$.

Comumente as mães são as principais perpetradoras de violências e castigos corporais contra os filhos ${ }^{28}$, em parte pelas normas sociais construídas para orientar o que se espera de homens e mulheres na condução da educação infantil e familiar. Apesar dos muitos avanços nas políticas públicas para reduzir as desigualdades entre mulheres e homens, a noção tradicional de gênero também pode ajudar na compreensão do papel da mulher como cuidadora e sua responsabilidade na gestão e cuidado da casa e dos filhos. Como visto neste estudo, são as mulheres, essencialmente em idade produtiva, as principais cuidadoras a abdicar do trabalho formal para o cuidado das crianças e adolescentes, imputando sobrecarga física e emocional e aumentando as chances de violência e resultados negativos contra as crianças $^{29-31}$.

Contudo, esta investigação traz uma nova forma de olhar para este fenômeno, uma vez que o cuidador-homem foi apontado como principal responsável pelos eventos de violência física grave (5,7 chances em relação as mulheres). Giffin $^{32}$ já havia refletido que o ambiente domiciliar idealizado como protetor e fraterno também tem sido o principal local para os abusos masculinos contra mulheres, crianças e idosos, e também o lugar privilegiado para a sua sistemática ocultação. Ademais, é importante problematizar que a maior parte das investigações sobre abusos físicos contra crianças têm sido realizadas com a população geral, não focalizando o grupo de crianças em situações crônicas. Outro ponto é o próprio envolvimento dos adolescentes na presente investigação, que por si só já diferencia a base populacional da maior parte das investigações realizadas.

Na contramão dos fatores que se associam a ocorrência de violências, foi observado um importante efeito protetor das violências físicas contra as crianças e adolescentes em famílias que estavam inscritas em programas governamentais de transferência de renda, reduzindo em $50 \%$ a ocorrência do evento. Os dois maiores programas são o Bolsa Família e os Benefícios de Prestação Continuada (BPC) são direcionados, respectivamente, a famílias pobres ou extremamente pobres, idosos e pessoas com deficiência ${ }^{33}$. Considerando a magnitude do Programa Bolsa Família, alguns estudos têm sido realizados apontando os efeitos benéficos da transferência de renda na alimentação ${ }^{34}$, tratamento de doenças infecciosas $^{35}$ e acesso aos serviços de saúde ${ }^{36}$.

Embora alguns autores já tenham associado programas de transferência de renda à redução de homicídios e hospitalizações provocadas pela violência ${ }^{37}$, este é um dos poucos estudos que mostra uma influência protetora contra a violência contra crianças e adolescentes com transtornos mentais. Essa redução pode ser explicada pelo controle dos setores de saúde e educação, por ser condição obrigatória para a continuidade do recebimento dos benefícios ${ }^{33}$. Além disso, é possível que os programas de transferência de renda estejam produzindo emancipação financeira às famílias cuja vida está diretamente dedicada ao cuidado de crianças e adolescentes com transtornos mentais graves, tornandose uma hipótese explicativa que merece maiores esclarecimentos em investigações futuras.

Embora este estudo contribua sobremaneira para o escrutínio dos fatores associados à violência física grave em um grupo de crianças e adolescentes com transtornos mentais graves, seus resultados devem ser interpretados à luz de suas limitações. A primeira refere-se ao escopo do estudo, que se limitou à análise de apenas uma unidade de saúde. Embora esta unidade de saúde seja uma referência para todas as crianças e adolescentes com transtornos mentais graves no município, é importante considerar que a rede de Atenção Básica também atende crianças e adolescentes em sofrimento psíquico, cenário não contemplado neste estudo. Com isso, os casos mais leves de transtornos mentais não foram recrutados, gerando um viés de seleção que pode ter aumentado as medidas de associação aqui encontradas.

Outro aspecto foi a forma de mensurar variáveis numéricas contínuas por meio de medidas categóricas, o que não permitiu observar nuances mais detalhadas do efeito do tempo sobre o desfecho, lacuna que merece ser abordada em análises futuras. Para isso se faz importante refletir sobre as formas de aferição destes eventos, já que o presente estudo encontrou predomínio de 10 anos no tempo de cuidado e de 40 horas na jornada semanal de cuidado. Refletir sobre novas formas de aferir esses eventos se faz necessário e urgente.

\section{CONCLUSÃO}

A violência física grave é prevalente entre crianças e adolescentes, especialmente devido à cultura de abusos que estrutura as relações de resolução de conflitos em muitas sociedades. Crianças e adolescentes com condições crônicas, principalmente transtornos mentais, apresentam maiores magnitudes de violência quando comparados a outros grupos. Este artigo apresenta uma análise dos fatores associados à ocorrência de violência física grave contra crianças e adolescentes com transtornos mentais, cenário ainda pouco explorado na literatura acadêmica. Identifica que o tempo 
dedicado ao cuidado e a jornada semanal de cuidado são fatores mais associados a estes eventos, trazendo a hipótese de que a sobrecarga dos cuidadores é um evento importante no fenômeno da violência contra crianças e adolescentes. Ademais, a idade, o sexo do cuidador e o tempo de diagnóstico também são fatores a serem considerados na cadeia produtora de violências contra este grupo populacional, aspectos que podem ser identificados pelos profissionais de saúde.

Deste modo, parece importante que a equipe de saúde mental desenvolva um conjunto de atitudes para a deteç̧ão precoce de riscos às crianças e adolescentes. A integração de familiares e cuidadores no desenvolvimento conjunto dos planos terapêuticos pode melhorar a abordagem das equipes de saúde, servindo como fator de proteção contra novos episódios de violência e de cuidado ampliado.

\section{REFERÊNCIAS}

1. Jaffee SR, Ambler A, Merrick M, Goldman-Mellor S, Odgers CL, Fisher HL, et al. Childhood maltreatment predicts poor economic and educational outcomes in the transition to adulthood. Am. J. Public Health [Internet]. 2018 [cited 2021 Jan 10]; 108:1142-7. https://doi.org/10.2105/AJPH.2018.304587.

2. Schaefer JD, Moffitt TE, Arseneault L, Danese A, Fisher HL, Houts R, et al. Adolescent Victimization and Early-Adult Psychopathology: Approaching Causal Inference Using a Longitudinal Twin Study to Rule Out Noncausal Explanations. Clin. Psychol. Sci. [Internet]. 2018 [cited 2021 Jan 10]; 6:352-71. https://doi.org/10.1177/2167702617741381.

3. Bordin IA, Duarte CS, Ribeiro WS, Paula CS, Coutinho ESF, Sourander A, et al. Violence and child mental health in Brazil: The Itaboraí Youth Study methods and findings. Int. J. Methods Psychiatr. Res. [Internet]. 2018 [cited 2021 Jan 10]; 27:1-15. https://doi.org/10.1002/mpr.1605.

4. Vanhalst J, Luyckx K, Goossens L. Experiencing Loneliness in Adolescence: A Matter of Individual Characteristics, Negative Peer Experiences, or Both? Soc. Dev. [Internet]. 2014 [cited 2021 Jan 10]; 23:100-18. DOI: https://doi.org/10.1111/sode.12019.

5. Ramos KÁ de A, Rafael R de MR, Penna LHG, Depret DG, Ribeiro LV, Carinhanha Jl. Sheltered adolescents' background of exposure to violence and distressful experiences. Rev. Bras. Enferm. [Internet]. 2020 [cited 2021 Jan 10]; 73:e20180714. DOI: https://doi.org/10.1590/0034-7167-2018-0714.

6. Krug EG, Dahlberg LL, Mercy JA, Zwi A, Lozano R, editors. World report on violence and health. Geneva: World Health Organization; 2002.

7. Straus MA. Measuring Intrafamily Conflict and Violence: The Conflict Tactics (CT) Scales. J. Marriage Fam. [Internet]. 1979 [cited 2021 Jan 10]; 41:75-88. DOI: https://doi.org/10.2307/351733.

8. Straus MA, Hamby SL, Finkelhor D, Moore DW, Runyan D. Identification of child maltreatment with the Parent-Child Conflict Tactics Scales: development and psychometric data for a national sample of American parents. Child Abuse Negl. [Internet]. 1998 [cited 2021 Jan 10]; 22:249-70. DOI: https://doi.org/10.1016/s0145-2134(97)00174-9.

9. Farias MS, Souza C da S, Carneseca EC, Passos ADC, Vieira EM. Caracterização das notificações de violência em crianças no município de Ribeirão Preto, São Paulo, no período 2006-2008. Epidemiol. e Serv. Saude Rev. Do Sist. Unico Saude Do Bras. [Internet]. 2016 [cited 2021 Jan 10]; 25:799-806. DOI: https://doi.org/10.5123/S1679-49742016000400013.

10. Santos SCF dos, Conceição LV da, Moura ATMS de, Rafael RM de R. Prevalence of family violence in children carrying chronic diseases admitted into paediatric specialty care units. Rev. Pediatr. SOPERJ [Internet]. 2016 [cited 2021 Jan 10]; 16:11-6. Available from: http://revistadepediatriasoperj.org.br/detalhe_artigo.asp?id=774.

11. Ribeiro IMP, Teixeira Ribeiro ÁS, Pratesi R, Gandolfi L. Prevalence of various forms of violence among school students. Acta Paul. Enferm. [Internet]. 2015 [cited 2021 Jan 10]; 28:54-9. DOI: https://doi.org/10.1590/1982-0194201500010.

12. Fidalgo TM, Sanchez ZM, Caetano SC, Andreoni S, Sanudo A, Chen Q, et al. Exposure to violence: Associations with psychiatric disorders in Brazilian youth. Rev. Bras. Psiquiatr. [Internet]. 2018 [cited 2021 Jan 10]; 40:277-83. DOI: https://doi.org/10.1590/1516-4446-2016-2122.

13. Pace GT, Lee SJ, Grogan-Kaylor A. Spanking and young children's socioemotional development in low- and middle-income countries. Child Abuse Negl. [Internet]. 2019 [cited 2021 Jan 10]; 88:84-95. DOI: https://doi.org/10.1016/j.chiabu.2018.11.003.

14. Marinho F, de Azeredo Passos VM, Carvalho Malta D, Barboza França E, Abreu DMX, Araújo VEM, et al. Burden of disease in Brazil, 1990-2016: a systematic subnational analysis for the Global Burden of Disease Study 2016. Lancet [Internet]. 2018 [cited 2021 Jan 10]; 392:760-75. DOI: https://doi.org/10.1016/S0140-6736(18)31221-2.

15. Jolly S, Griffith KA, DeCastro R, Stewart A, Ubel P, Jagsi R. Gender differences in time spent on parenting and domestic responsibilities by high-achieving young physician-researchers. Ann. Intern. Med. [Internet]. 2014 [cited 2021 Jan 10]; 160:34453. DOI: https://doi.org/10.7326/M13-0974.

16. Instituto Brasileiro de Geografia e Estatística (IBGE). Pesquisa Nacional por Amostra de Domicílios: questionário da pesquisa. Rio de Janeiro: IBGE; 2011.

17. Associação Brasileira de Empresas de Pesquisa (ABEP). Critérios de Classificação Econômica Brasil. Rio de Janeiro: ABEP; 2016.

18. Reichenheim ME, Moraes CL. Adaptation of the Parent-Child Conflict Tactics Scales (CTSPC), an instrument used to identify parental violenc. Cad. Saúde Pública [Internet]. 2003; 19:1701-12. DOI: https://doi.org/10.1590/S0102-311X2003000600014.

19. StataCorp. Stata Statistical Software: Release 152017.

20. Jaudes PK, Mackey-Bilaver L. Do chronic conditions increase young children's risk of being maltreated? Child Abuse Negl. [Internet]. 2008 [cited 2021 Jan 10]; 32:671-81. DOI: https://doi.org/10.1016/j.chiabu.2007.08.007. 
21. Miller GF, Chiang L, Hollis N. Economics and violence against children, findings from the Violence Against Children Survey in Nigeria. Child Abuse Negl. [Internet]. 2018 [cited 2021 Jan 10]; 85:9-16. DOI: https://doi.org/10.1016/j.chiabu.2018.08.021.

22. Sitnick SL, Shaw DS, Weaver CM, Shelleby EC, Choe DE, Reuben JD, et al. Early Childhood Predictors of Severe Youth Violence in Low-Income Male Adolescents. Child Dev. [Internet]. 2017 [cited 2021 Jan 10]; 88:27-40. DOI: https://doi.org/10.1111/cdev.12680.

23. Bai G, Herten MH, Landgraf JM, Korfage IJ, Raat H. Childhood chronic conditions and health-related quality of life: Findings from a large population-based study. PLoS One [Internet]. 2017 [cited 2021 Jan 10]; 12:e0178539-e0178539. DOI: https://doi.org/10.1371/journal.pone.0178539.

24. Coutinho VM, Queiroga BAM de, Souza RC de. Attachment style in children with chronic diseases: A comprehensive review. Rev. Paul. Pediatr. [Internet]. 2020 [cited 2021 Jan 10]; 38:e2018308-e2018308. DOI: https://doi.org/10.1590/19840462/2020/38/2018308.

25. Adib-Hajbaghery M, Ahmadi B. Caregiver Burden and Its Associated Factors in Caregivers of Children and Adolescents with Chronic Conditions. Int. J. Community Based Nurs Midwifery [Internet]. 2019 [cited 2021 Jan 10]; 7:258-69. DOI: https://doi.org/10.30476/IJCBNM.2019.73893.0.

26. Hanson CL, Crandall A, Barnes MD, Magnusson B, Novilla MLB, King J. Family-Focused Public Health: Supporting Homes and Families in Policy and Practice. Front Public Heal. [Internet]. 2019 [cited 2021 Jan 10]; 7:59. DOI: https://doi.org/10.3389/fpubh.2019.00059.

27. Lansford JE, Godwin J, Uribe Tirado LM, Zelli A, Al-Hassan SM, Bacchini D, et al. Individual, family, and culture level contributions to child physical abuse and neglect: A longitudinal study in nine countries. Dev. Psychopathol. [Internet]. 2015 [cited 2021 Jan 10]; 27:1417-28. DOI: https://doi.org/10.1017/S095457941500084X.

28. Moura ATMS, Moraes CL, Reichenheim ME. Detection of child abuse: missed opportunities in emergency rooms in Rio de Janeiro, Brazil. Cad. Saude Publica [Internet]. 2008 [cited 2021 Jan 10]; 24:2926-36. DOI: https://doi.org/10.1590/S0102$311 X 2008001200022$.

29. Rafael RMR, Moura ATMS de. Severe intimate partner physical violence as a risk factor for inadequate cervical cancer screening. Cad. Saude Publica [Internet]. 2017 [cited 2021 Jan 10]; 33:e00074216. DOI: https://doi.org/10.1590/0102$311 \times 00074216$.

30. Buonomo I, Fiorilli C, Romano L, Benevene P. The Roles of Work-Life Conflict and Gender in the Relationship between Workplace Bullying and Personal Burnout. A Study on Italian School Principals. Int. J. Environ. Res. Public Health [Internet]. 2020 [cited 2021 Jan 10]; 17:8745. DOI: https://doi.org/10.3390/ijerph17238745.

31. Carlson C, Namy S, Norcini Pala A, Wainberg ML, Michau L, Nakuti J, et al. Violence against children and intimate partner violence against women: overlap and common contributing factors among caregiver-adolescent dyads. BMC Public Health [Internet].2020 [cited 2021 Jan 10]; 20:124. DOI: https://doi.org/10.1186/s12889-019-8115-0.

32. Giffin K. Gender violence, sexuality, and health. Cad. Saude Publica [Internet]. 1994 [cited 2021 Jan 10]; 10:S146-55. DOI: https://doi.org/10.1590/s0102-311×1994000500010.

33. Denes G, Komatsu BK, Menezes-Filho N. Uma avaliação dos impactos macroeconômicos e sociais de programas de transferência de renda nos municípios Brasileiros. Rev. Bras. Econ. [Internet]. 2018 [cited 2021 Jan 10]; 72:292-312. Available from: https://www.scielo.br/j/rbe/a/z588jJkmH7YrWg9PrgMJZpD/?format=pdf\&lang=pt.

34. Martins APB, Monteiro CA. Impact of the Bolsa Família program on food availability of low-income Brazilian families: a quasi experimental study. BMC Public Health [Internet]. 2016 [cited 2021 Jan 10]; 16:827. DOI: https://doi.org/10.1186/s12889-0163486-y.

35. Oliosi JGN, Reis-Santos B, Locatelli RL, Sales CMM, da Silva Filho WG, da Silva KC, et al. Effect of the Bolsa Familia Programme on the outcome of tuberculosis treatment: a prospective cohort study. Lancet Glob Heal. [Internet]. 2019 [cited 2021 Jan 10]; 7:e219-26. DOI: https://doi.org/10.1016/S2214-109X(18)30478-9.

36. Shei A, Costa F, Reis MG, Ko Al. The impact of Brazil's Bolsa Família conditional cash transfer program on children's health care utilization and health outcomes. BMC Int. Health Hum. Rights [Internet]. 2014 [cited 2021 Jan 10]; 14:10. DOI: https://doi.org/10.1186/1472-698X-14-10.

37. Machado DB, Rodrigues LC, Rasella D, Lima Barreto M, Araya R. Conditional cash transfer programme: Impact on homicide rates and hospitalisations from violence in Brazil. PLoS One [Internet]. 2018 [cited 2021 Jan 10]; 13:e0208925-e0208925. DOI: https://doi.org/10.1371/journal.pone.0208925. 